\title{
Review on liver steatosis and its impact on liver transplantation
}

\begin{abstract}
The lack of available organs for liver transplantation (LT) associated with the increased death rates among patients on the waiting lists for LT has triggered the use of so-called extended criteria donor (ECD) grafts. Among the wide range of these ECD livers, hepatic steatosis is one of the most frequent disorders, which is mostly related to an increasing prevalence of non-alcoholic fatty liver disease (NAFLD) NAFLD is increasingly significant in healthy individuals for its high worldwide prevalence, an association with the metabolic syndrome, such as insulin resistance, diabetes, dyslipidemia and obesity, and an association with liver-related morbidity and mortality. Using the ECD grafts especially those with NAFLD will markedly increase the donor pool for LT. Several approaches, including long-term and shortterm measures, were identified for the management of NAFLD.
\end{abstract}

Keywords: steatosis, liver transplantation, fatty liver, ischemia, reperfusion injury
Volume 3 Issue 4 - 2017

\author{
Emad Ali Ahmed, ${ }^{1,2}$ Ashraf Mohammad El \\ Badry,' Asem Elsani Ali Hassan,' Alaa Ahmed \\ Redwan,' Marco Vivarelli² \\ 'Department of General Surgery, University of Sohag, Egypt \\ ${ }^{2}$ Department of Experimental and Clinical Medicine, Polytechnic \\ University of Marche, Italy
}

\begin{abstract}
Correspondence: Emad Ali Ahmed, Hepatobiliary and Pancreatic Surgery Unit, Department of General Surgery, Sohag University Hospitals, Sohag University, Sohag, Egypt, Tel 002010
\end{abstract} 6163 2696, Fax 4609304, Email dr.emadali@yahoo.com

Received: August 24, 2017| Published:September 21, 2017
Abbreviations: ALT, alanine amino transferases; AST, aspartate amino transferases; AUROC, area under the receiver operating characteristic curve; BMI, body mass index; CIT, cold ischemia time; $\mathrm{CT}$, computed tomography; D-MELD, donor age multiplied with the recipient model for end-stage liver disease; ECD, extended criteria donor; EGD, early graft dysfunction; ELF, enhanced liver fibrosis; ESLD, end-stage liver disease; FA, fatty acids; FIB-4, fibrosis-4; FLI, fatty liver index; FLIP, fatty liver inhibition of progression; GGT, gamma-glutamyl transpeptidase; HCC, hepatocellular carcinoma; HCV, hepatitis c virus; IP, ischemic preconditioning; IR, insulin resistance; $\mathrm{I} / \mathrm{R}$, ischemia reperfusion; LDLT, living donor liver transplantation; LSM, liver stiffness measurement; LT, liver transplantation; MaS, macrosteatosis; MetS, metabolic syndrome; MiS, microsteatosis; MELD, model for end-stage liver disease; MR, magnetic resonance; NAFL, non-alcoholic fatty liver; NAFLD, nonalcoholic fatty liver disease; NAS, nafld activity score; NASH, nonalcoholic steatohepatitis; NFS, nafld fibrosis score; NPV, Negative Predictive Value; PNF, primary non-function; PPV, positive predictive value; ROS, reactive oxygen species; T2DM. type 2 diabetes mellitus; Tnf- $\alpha$, tumor necrosing factor A; UNOS, united network of organ sharing; WIT, warm ischemia time

\section{Introduction}

As organs shortage was anticipated as early as 1987, the development of what is called "marginal donors" was crucial. ${ }^{1}$ A marginal graft could be defined as an organ with an increased risk for poor function or failure that may subject the recipient to higher risks of morbidity or mortality. However, there is no consensus about factors that define a graft as marginal and should be excluded from the use because of unacceptable risk to the recipient. Therefore, the decision to transplant a specific organ depends on the judgment of the transplant surgeon and consideration of the specific recipient. ${ }^{2}$ Broadly there are two categories of marginal grafts. Firstly, there are grafts which carry a high risk of technical complications and/or impaired function, including the steatotic livers, non-heart beating donors, elderly donors, split livers, donors with high inotrope requirement or long ischaemia times. Secondly, grafts will be considered marginal if they carry a risk of transmission of infection and/or malignancy to the recipient. ${ }^{2}$ Among the wide range of these ECD livers, hepatic steatosis is one of the most frequent disorders, which is mostly related to an increasing prevalence of fatty liver diseases. ${ }^{3}$

\section{Definition}

Fatty liver is defined as the fat accumulation of at least 5\% of liver weight in the absence of other etiologies of liver disease or use of steatogenic medication. ${ }^{4}$ Traditionally, fatty disorders of the liver have been classified into alcoholic and non-alcoholic. NAFLD includes a spectrum of diseases ranging from pure steatosis (nonalcoholic fatty liver [NAFL]) to NASH. ${ }^{5}$ NAFL is defined as the presence of hepatic steatosis with no evidence of hepatocellular injury in the form of ballooning of the hepatocytes. NASH is defined as the presence of hepatic steatosis and inflammation with hepatocyte injury (ballooning) with or without fibrosis. ${ }^{6}$ NAFL is further subdivided into Macrosteatosis (MaS) and Microsteatosis (MiS). MaS are characterized by a single, bulky fat vacuole in hepatocytes, displacing the nucleus to the edge of the cell. In MiS, the cytoplasm of the hepatocytes contains tiny lipid vesicles without nuclear dislocation. ${ }^{7}$

\section{Epidemiology}

Incidence and prevalence: NAFLD is the most common chronic liver disease in the general population, posing an enormous burden on public healthcare costs. Its prevalence has increased as more patients develop a sedentary lifestyle, metabolic syndrome (MetS), and obesity. It is correlated with many factors, including body mass index (BMI), fat distribution, race, ethnicity, and sex. ${ }^{8}$ Moreover, NAFLD occurrence increases with age and are generally higher in men than in pre-menopausal women. It also differs according to ethnicity, affecting $45 \%$ of Hispanic people, $33 \%$ of Caucasian and $24 \%$ of African-Americans in the USA, as well as increases in South Asians and United Kingdom. ${ }^{9}$ In a recent meta-analysis, the global prevalence of NAFLD was estimated to be $25 \%$, which is very similar to that of the MetS. Prevalence estimates averaged $32 \%$ in the Middle East, $30 \%$ in South America, 27\% in Asia, 24\% in Europe, $21 \%$ in North America and $13 \%$ in Africa. ${ }^{10}$ 
Natural history: While pure steatosis is not associated with increased mortality in long-term follow-up studies, individuals with NASH have a reduced life expectancy. Recent evidence suggests that in addition to liver-related death (due to end-stage liver disease, portal hypertension and hepatocellular carcinoma (HCC), patients are exposed to increased frequency of cardiovascular diseases leading to excess mortality. Patients with either uncomplicated steatosis or NASH appear to be at risk of developing the full-blown MetS or its individual components, particularly type II diabetes mellitus (T2DM) and impaired fasting glucose. Progression to fibrosis has been demonstrated histologically in up to $30 \%$ of cases, but apparent regression may occur. Cirrhosis develops in up to $35 \%$ of those cases with a risk of liver failure or HCC. ${ }^{11}$ Therefore, the increasing prevalence of NAFLD is expected to raise the number of liver transplantation (LT) candidates in the near future, and possibly become the most common indication for LT. ${ }^{3}$

Risk factors: Given the tight association with MetS, the whole spectrum of NAFLD mostly occurs in patients with obesity (60-95\%), T2DM (28-55\%), and hyperlipidemia (27-92\%). In a study of patients undergoing bariatric surgery, NAFLD was found in up to $95 \%$, with fibrosis in $74 \%$, and mild necroinflammation in $24 \%$ of cases. The pattern of fat distribution is more important than BMI, and visceral fat has been associated with severity of inflammation and fibrosis. Abnormal glucose regulation per se carries an increased risk for NAFLD, whose prevalence rises from $27 \%$ in subjects with normal basal glucose to $43 \%$ in impaired fasting glucose and $62 \%$ in T2DM.

\section{Pathogenesis}

Although the exact pathogenesis of NAFLD remains poorly understood, there were different hypotheses involved in NAFLD development. Hepatic lipid content is regulated by a balance between hepatic lipid uptake, synthesis, oxidation, and export. Most of the triglycerides are re-esterified fatty acids (FA) that originate from adipose tissue lipolysis and come into the liver. ${ }^{12}$ The adipose tissue lipolysis provides approximately $60 \%$ of the FAs used for hepatic triglyceride synthesis. The rest of the hepatic FAs come from de novo lipogenesis within the liver $(25 \%)$ and dietary intake $(15 \%) .{ }^{13}$ Steatosis reflects a net retention of fat within hepatocytes and results from an imbalance between uptake of fat and its oxidation and export. The most consistent pathogenic factor is insulin resistance (IR), leading to enhanced lipolysis which in turn increases circulating free FAs and their uptake by the liver. Fat accumulating in the liver has several effects:

a. Up regulation of apoptosis

b. Upregulation of tumor necrosing factor $\alpha(\mathrm{TNF}-\alpha)$ which is pro-steatotic and pro-inflammatory

c. Mitochondrial dysfunction presumably increasing reactive oxygen species (ROS) and provoking lipid peroxidation of cell membranes

d. Induction of cytochrome P450 which generates ROS

e. Induction of pro-inflammatory genes such as TNF- $\alpha$ and cyclooxygenase enzyme- 2 which induce additional inflammatory mediators which are also pro-fibrotic.

The net effect of the above is apoptosis, necro-inflammation, and fibrosis. ${ }^{14}$ Moreover, excess hepatic fat results in increased very low-density lipoprotein secretion and some of the serum lipid abnormalities noted in MetS and NAFLD, including hypertri- glyceridemia, decreased high-density lipoprotein, and higher lowdensity lipoprotein. ${ }^{15}$ Hepatic fibrosis is promoted by steatosis even in the absence of liver cell injury. Adipokines, hormones secreted by adipocytes, appear to be important regulators of hepatic fibrosis. Leptin, which is increased in the MetS, promotes fibrosis and induces pro-inflammatory cytokines while adiponectin, which is decreased in MetS, inhibits stellate cell activation. The net effect of increased leptin and decreased adiponectin is pro-inflammatory and pro-fibrotic mediators. ${ }^{14}$ Another prevailing hypothesis considered that several insults or "hits" are involved in causing progressive liver injury with the initial hit, MaS results. IR most likely plays a central role in the net retention of lipids, particularly triglycerides, within the hepatocytes. This is thought to result from decreased disposal of FAs due to impaired mitochondrial $\beta$-oxidation. The second hit is generally attributed to oxidative stress, which causes peroxidation of lipids in the hepatocyte membrane with cytokine production and is in large part responsible for the progression from simple steatosis to NASH to liver cirrhosis. ${ }^{16,17}$ Additional theories, such as the multiplehit hypothesis, suggest other factors including clinical conditions that may also involve in NAFLD. ${ }^{18}$ Elevated circulating levels of pro-inflammatory cytokines and reduced levels of anti-inflammatory factors cause a chronic low-grade inflammatory state that is recognized as an important pathogenic mechanism of NAFLD. Among clinical associations, diabetes and MetS, advanced age ( $>45$ years), Hispanic ethnicity, female sex, and obesity (BMI $>30)$ are all related to more aggressive liver histology but without a clear distinction of the cause and effect. ${ }^{14,19,20}$

New insights have been recently provided regarding the fat composition in the steatotic livers, particularly the omega-3 and omega-6 FA ratio. In this context, omega-3 FAs down regulate the sterol regulatory element binding protein-1 which enhances hepatic triglyceride accumulation. Moreover, omega-3 FAs upregulate peroxisomal proliferator-activated receptor a, which stimulates lipid degradation. Conversely, these actions can be offset by excessive intake of omega-6 Fas. ${ }^{3}$

\section{Diagnosis}

Clinical presentation: Most patients with NAFLD (48-100\%) are asymptomatic. The liver disease is often discovered incidentally during the routine examination. When symptoms occur, they are usually nonspecific as vague right upper quadrant abdominal pain, fatigue, and malaise. Rarely, pruritus, anorexia, and nausea may develop. Jaundice, abdominal distension (ascites), gastrointestinal bleeding, and confusion (encephalopathy) are all indicative of advanced liver disease (decompensated cirrhosis), occurring late in the course. There are no pathognomonic signs of NAFLD. Obesity is the most common abnormality on physical examination, occurring in $30-100 \%$ while hepatomegaly has been reported in up to $75 \%$ of patients. ${ }^{21}$

\section{Non-invasive tests}

Laboratory investigations: Mild to moderate elevation of serum alanine (ALT) and aspartate aminotransferases (AST) and/or alkaline phosphatase and gamma-glutamyl transpeptidase (GGT) is the most common laboratory abnormality that could be found in patients with NAFLD. ${ }^{22}$ Unlike those with alcohol-induced steatohepatitis, who typically manifest disproportionate increases in the AST level relative to the ALT level, patients with NAFLD usually have an AST/ALT ratio $<1$. The AST/ALT ratio tends to increase with the development of cirrhosis, thus losing its diagnostic accuracy. Elevated serum lipid 
profiles and glucose concentrations are also common in NAFLD patients, reported in 25 to $75 \%$ of cases. A small percentage of patients with NAFLD may have a low-titer $(\leq 1: 320)$ antinuclear antibody (ANA) positivity. ${ }^{21}$

\section{Clinico-laboratory predictor models}

In order to restrict the large number of individuals potentially susceptible to undergo a liver biopsy on the account of suspected NAFLD, clinicians have evaluated the utility of several indices predictors for diagnosis of steatosis, NASH and fibrosis in NAFLD patients, including: ${ }^{11,23}$

Fatty Liver Index (FLI): this algorithm was based on four markers; BMI, waist circumference $(\mathrm{cm})$, triglyceride $(\mathrm{mg} / \mathrm{dL})$ and GGT $(\mathrm{U} / \mathrm{L})$, which is confirmed to accurately identify NAFLD. This index has been used in population studies and has achieved an area under the receiver operating characteristic curve ("AUROC", which measures the accuracy of the test, with the values close to 1 represent a perfect test) of 0.84 in detecting fatty liver. FLI provides a score out of 100 , indicating that a score $<30$ can rule out and a score $\geq 60$ to rule in hepatic steatosis. ${ }^{24}$

The hair (Hypertension, ALT, IR) model: is an assessment of morbidly obese patients (BMI $>35)$, to develop NASH, by assigning a point each for the presence of hypertension, elevated ALT ( $>40 \mathrm{U} / \mathrm{L})$ and IR Index $>5$. A HAIR score of $\geq 2$ was $80 \%$ sensitive and $89 \%$ specific for NASH. ${ }^{25}$

The NASH clinical scoring system: scores for DM, hypertension, elevated AST ( $\geq 27 \mathrm{IU} / \mathrm{L})$, elevated ALT ( $\geq 27 \mathrm{IU} / \mathrm{L})$, sleep apnea and race) is also used for morbidly obese patients, and had an AUROC of 0.8 , sensitivity $22 \%$ and specificity $99 \%$. This system has some limitations as the normal reference ranges were not defined and patients with NASH may have serum transaminases within the normal reference range. ${ }^{26}$

The NASH predictive index incorporates age, BMI, homeostatic model assessment (HOMA)-IR, AST, ALT and sex (female), and produced an AUROC of 0.86 , sensitivity $56 \%$, and specificity $100 \% .{ }^{27}$

The NASH test: uses a combination of 13 clinical and biochemical variables including age, gender, and weight, height, and serum levels of cholesterol, triglycerides, $\alpha$-2macroglobulin, apolipoprotein A1, haptoglobin, GGT, ALT, AST, and bilirubin. The AUROC for diagnosing NASH as determined by liver biopsy was 0.78 and by the test 0.79 , with a sensitivity of $29 \%$, specificity of $98 \%$, positive predictive value (PPV) of $91 \%$, and negative predictive value (NPV) of $71 \%$. The NASH Test has been validated from different centers as a simple non-invasive test for diagnosis of NASH. ${ }^{28}$

The AAR (AST: ALT Ratio) model: The AST-to-ALT ratio is the simplest predictive model for fibrosis. ALT is typically higher than AST in NAFLD; however, having an AAR $>1$ is suggestive of the presence of advanced fibrosis. AAR has a good negative predictive value (NPV) to rule out advanced fibrosis. ${ }^{29}$

The APRI (AST and Platelet Ratio index): is calculated using the AST elevation (AST level divided by the upper limit of normal for the laboratory) and the platelet count per $\mathrm{mm}^{3}$ divided by 1000 . APRI has been designed to detect significant fibrosis and cirrhosis and suggested cutoffs for significant fibrosis are $>1$ and for cirrhosis $>1.5$. In a recent study involving 358 patients with biopsy-proven NAFLD, the AUROC, sensitivity, and specificity of APRI with a cutoff $>1$ for significant fibrosis were, respectively, $0.67,30$, and $93 \%$ and a cutoff $>1.5$ for cirrhosis were $0.94,76$, and $72 \%$, respectively. ${ }^{30}$

NAFLD fibrosis score (NFS): this score is a widely accepted tool in excluding advanced fibrosis evaluates six variables; age, hyperglycemia, BMI, platelet count, albumin, and AST/ALT ratio. It has AUROC 0.82 , sensitivity $82 \%$, specificity $77 \%$, PPV $56 \%$, and NPV $93 \%$. This score is used to classify the probability of fibrosis as $<-1.5$ for low probability and $>-1.5$ to $<0.67$ for intermediate probability and $>0.67$ for high probability. ${ }^{31}$ It has been acknowledged by the current NAFLD guidelines as a clinically useful tool for identifying advanced fibrosis in NAFLD. Another advantage of the NFS is its ability to provide prognostic information and identify patients with NAFLD who are at increased risk for liver-related complications (such as cites and gastroesophageal varices) or death. A major limitation of this score is that a significant percentage (20-58\%) of patients fall between the two proposed cutoff values and therefore, they have an indeterminate score. ${ }^{32}$

Fibrometer: This tool uses age, weight, fasting glucose, AST, ALT, ferritin and platelet count to diagnose significant fibrosis. ${ }^{33}$ The Fibro meter provides the probability of significant fibrosis and the percentage of hepatic fibrosis. Calès et al ${ }^{34}$ compared the Fibro Meter to NFS and found that the Fibro Meter provides a more reliable diagnosis of significant fibrosis than the NFS. This tool can be used to confirm or disconfirm advanced fibrosis in NAFLD patients. ${ }^{34}$

The BARD (BMI, AST, ALT Ratio and Diabetes) score: combines clinical features of BMI and the presence or absence of diabetes, with the AST/ALT ratio. It has been designed to detect advanced fibrosis. Using a cutoff point $>2$, the sensitivity for the detection of advanced fibrosis was between 86.8 and $100 \%$, while the specificity ranged from 32.5 to $34.7 \%$ in different studies. The main limitation of the BARD score is its low specificity, giving a high false positivity, based on the overestimation of BMI and presence of diabetes. ${ }^{35}$

The BAAT (BMI, Age, ALT, Triglyceride) score: including age $\left(>50\right.$ years), BMI $\left(>28 \mathrm{~kg} / \mathrm{m}^{2}\right)$, ALT $(>2 \times \mathrm{N})$, and serum triglycerides $(>1.7 \mathrm{mmol} / \mathrm{L})$. Each variable received a score of 0 or 1 . Although a total score of 0 had sensitivity close to $100 \%$ and a specificity of $47 \%$, a high total score of 4 gave a sensitivity of $14 \%$ and a specificity of $100 \%$ for detection of septal fibrosis to assess advanced liver fibrosis in overweight patients. For a cutoff BAAT score of 2, the sensitivity, specificity, PPV, and NPV were $71,80,61$, and $86 \%$, with an AUROC of $0.83 .{ }^{36}$

Fibrosis-4 (FIB-4) index: Although the FIB-4 index was originally developed to stage liver fibrosis in patients with hepatitis $\mathrm{C}$ infection, it has shown promising results in patients with NAFLD. This algorithm utilizes platelet count, ALT, AST, and age with dual cutoff values. For diagnosing advanced fibrosis, the cutoff is 1.45 , giving an AUROC of 0.87 with a sensitivity of $74 \%$ and a specificity of $71 \%$, resulting in a PPV of $22 \%$ and NPV of $73 \%$. The upper cutoff is 3.25 , giving an AUROC of 0.88 with a sensitivity of $26 \%$ and specificity of $98 \%$ and providing PPV of $75 \%$ and NPV of $85 \%$. Utilizing the two cutoffs is clinically useful since the below the lower cutoff is good for excluding advanced fibrosis and patients above the upper cutoff are more likely to have advanced fibrosis. ${ }^{37}$

Fibro test: is another panel that predicts the presence of fibrosis by using five biomarkers (haptoglobin, $\alpha 2$-macroglobulin, apolipoprotein A1, total bilirubin, and GGT). The diagnostic value of Fibro Test was assessed in a large cohort of NAFLD patients and demonstrated that 
it can reliably predict advanced fibrosis with an AUROC of $0.88 .^{38}$ However, caution should be exercised when interpreting the results of this test in those with Gilbert's syndrome and cholestasis and also in acute inflammation as these conditions will increase bilirubin and haptoglobin level, respectively. FibroTest is available commercially in the USA as a part of the NASH-FibroSURE® (LabCorp, NC). ${ }^{32}$

The Enhanced Liver Fibrosis (ELF) Test: ELF test is an algorithm that includes hyaluronic acid, amino-terminal propeptide of type III procollagen, and tissue inhibitor of metalloproteinase 1. It had a good performance in distinguishing advanced fibrosis with a cutoff 10.3 with an AUROC of 0.90, but lower AUROC of 0.82 for significant fibrosis with a cutoff 9.3 and 0.76 for the absence of fibrosis with a cutoff $<8.5$. Interestingly, the ELF test was shown to be a good predictor of liver-related morbidity and mortality in a group of patients with a chronic liver disease, including 44 patients with NAFLD, suggesting that it might be a promising prognostic tool. ${ }^{39}$

Palekar et al. ${ }^{40}$ Model: a clinical model to distinguish NASH from simple steatosis by combining 5 different variables including age ( $\geq 50$ years), female gender, AST ( $\geq 45 \mathrm{U} / \mathrm{L})$, body mass index (BMI $\left.\geq 30 \mathrm{~kg} / \mathrm{m}^{2}\right)$, and AST/ALT ratio $(\geq 0.8)$, and serum hyaluronic acid $(\geq 55 \mathrm{mg} / \mathrm{L})$. The AUC for this model was 0.76 . The presence of 3 or more of these factors had a sensitivity, specificity, PPV, and NPV for NASH diagnosis of $74 \%, 66 \%, 68 \%$, and $71 \%$, respectively. ${ }^{40}$

\section{Imaging study}

Several noninvasive imaging techniques can provide semiquantitative data in NAFLD with variable accuracy measures. These techniques include:

Ultrasonography (US): it can identify hepatic steatosis and have been advocated as a diagnostic test for NAFLD. US have an overall sensitivity of $89 \%$ with $93 \%$ specificity, ${ }^{41}$ but markedly decreased in those with the mild disease when the amount of fat in the liver is less than $30 \%$ or in the presence of fibrosis. ${ }^{42}$ The ultrasonic feature in NAFLD is the "bright" liver with increased parenchymal echotexture compared with the kidneys and associated with vascular blurring. Areas of focal sparring can give the appearance of metastases. The PPV and NPV of ultrasound in patients with abnormal liver chemistries and other causes of liver disease ruled out are $96 \%$ and $19 \%$ respectively. ${ }^{14}$ On the basis of increasing echogenicity of the liver parenchyma compared with that of the right kidney and decreased visualization of the diaphragm and intrahepatic vessel borders, steatosis in each patient was graded as none (normal US liver structure), mild (slight increase of echogenicity, normal visualization), moderate (diffuse increase of echogenicity, slight impaired visualization), or severe (marked increase of echogenicity, poor or no visualization). ${ }^{43} \mathrm{~A}$ review conducted by Festi et al. ${ }^{44}$ concluded that ultrasonography should be used as a first-line diagnostic tool because of its evaluation of liver steatosis and other abdominal organs. ${ }^{44}$

Computed Tomography (CT): Fatty infiltration of the liver produces a low-density hepatic parenchyma on CT scanning. In a direct comparison of CT with the US, US were found to be more sensitive in detecting fatty change. However, when fatty change is patchy or focal or when a semi-quantitative assessment is required, CT scan and MRI are superior to US. ${ }^{21}$

Magnetic Resonance (MRI) Imaging: T1-weighted dual-echo MR imaging and MR spectroscopy are superior to US and CT scans and strongly correlate with histopathologic steatosis assessment. In contrast to US and CT, T1-weighted dual-echo MR imaging and MR spectroscopy are better able to depict differences across steatosis grades. These modalities showed the best diagnostic accuracies. ${ }^{45}$

Fibro scan (Transient elastography): it is another noninvasive method to assess liver fibrosis. This method measures liver stiffness, which was originally designed for the hepatitis $\mathrm{C}$ population, but is now being used in the NAFLD population. The Fibro Scan sends a pulse through the skin, which is circulated through the liver. The velocity of the wave, which is correlated with liver stiffness, is measured by ultrasound. The stiffer the liver the greater the degree of fibrosis. The liver stiffness measurement is used to assess the current stage of liver fibrosis. The cutoffs are 4.85, 7.38, 9.28, 13.33 and $25.34 \mathrm{kPa}$ which represent stages, 0 (no steatosis), 1(perivenular and/ or perisinusoidal fibrosis), 2(combined pericellular portal fibrosis), 3 (septal fibrosis) and 4 (cirrhosis). ${ }^{23}$ Transient elastography has been validated as a screening tool for advanced fibrosis in NAFLD, with a high NPV of $97 \%$ however, it cannot be used to rule out inflammation of the liver. ${ }^{46}$ Overall, these noninvasive measurements, including the clinic-laboratory predictor models and imaging techniques to assess NAFLD patients should be used prior to a liver biopsy as they pose minimal risk to the patient. However, liver biopsy should be considered in patients when these noninvasive tests are inconclusive, or the patients have risk factors associated with advanced fibrosis, such as age $>50$ years, the presence of diabetes, morbid obesity or metabolic syndrome..$^{23}$

Invasive test (Liver biopsy): Despite the utility of several imaging modalities in the diagnosis of diffuse fatty disorders of the liver, none is sufficiently sensitive to detect fibrosis or cirrhosis. Therefore, liver biopsy is considered as the gold standard for diagnosis in such conditions. ${ }^{21}$ More recently, the NASH clinical research network (NASH-CRN) stated that NASH was "established by the presence of a characteristic pattern of steatosis, inflammation, and hepatocellular ballooning on liver biopsies in the absence of significant alcohol consumption," thereby eliminating sinusoidal fibrosis from the definition. A distinction between no NASH, borderline, and definite NASH has also been proposed, underlining current difficulty in establishing a clear distinction between what constitutes NASH and what does not. ${ }^{47}$ Despite the availability of different lipid stains such as Sudan III, toluidine blue, and Oil Red O, frozen-section H\&E staining remains the most commonly used technique worldwide due to time constraints. ${ }^{7}$ Steatosis is typically characterized quantitatively and qualitatively. The quantitative evaluation is based on the percentage of hepatocytes containing cytoplasmic fat inclusions. There are many quantitative systems that grade liver steatosis. Ploeg et al.$^{48}$ classified steatosis into mild, moderate and severe if $\geq 5 \%$ and $<30 \%, \geq 30 \%$ and $\leq 60 \%$, or $\geq 60 \%$ of hepatocytes contain fat vacuoles within the cytoplasm, respectively. ${ }^{48}$ Brunt et al. ${ }^{49}$ described the mild, moderate and severe degrees of steatosis if between $5 \%$ and $33 \%$, between $33 \%$ and $66 \%$, or more than $66 \%$, respectively. ${ }^{49}$ In the clinical setting of LT, Ploeg et al. ${ }^{48}$ description is sounder when studying graft steatosis. As described before, fatty infiltration is separated qualitatively into two categories, MaS and MiS. MaS are characterized by a single, bulky fat vacuole in hepatocytes, displacing the nucleus to the edge of the cell. In MiS, the cytoplasm of the hepatocytes contains tiny lipid vesicles without nuclear dislocation. MiS are usually encountered in mitochondrial disruption following acute viral, toxin- or druginduced injury, sepsis, and in some metabolic disorders. However, the quantitative methods provide information only on the total amount of fat, omitting any data on the chemical composition of hepatic 
lipids, novel objective tools such as measurement of the omega- 6 and omega-3 FA levels in liver biopsy samples, may help prediction of the magnitude of reperfusion injury. ${ }^{3}$ Importantly, other histopathological features should be carefully assessed in the presence of steatosis including inflammation, fibrosis, and ballooning degeneration. Different systems were developed for discrimination between simple steatosis and NASH, including:

The NAFLD Activity Score (NAS): Kleiner et al. ${ }^{50}$ proposed the NAS which specifically includes only features of active injury that are potentially reversible in the short term. The score is defined as the unweighted sum of the scores for steatosis (0-3), lobular inflammation (0-3), and ballooning (0-2); thus, ranging from 0 to 8 . Fibrosis, which is both less reversible and generally thought to be a result of disease activity, is not included as a component of the activity score. The separation of fibrosis from other features of activity is an accepted paradigm for staging and grading for both NASH and chronic hepatitis. Cases with NAS of 0 to 2 were largely considered not diagnostic of steatohepatitis; on the other hand, most cases with scores of $\geq 5$ were diagnosed as steatohepatitis. Cases with activity scores of 3 and 4 were divided almost evenly between the 3 diagnostic categories. ${ }^{50}$

The histopathological Fatty Liver Inhibition of Progression (FLIP) algorithm: Bedossa et al. ${ }^{47}$ defined NAFLD by the presence of steatosis in $\geq 5 \%$ of hepatocytes, and NASH by the presence, in addition, of hepatocellular ballooning of any degree and lobular inflammatory infiltrates of any amount. Therefore, steatosis was used as the criteria for entry into the algorithm weighted by hepatocellular ballooning and lobular inflammation. In the algorithm tree, all cases with at least grade 1 steatosis were diagnosed NAFLD independently of other criteria, whereas when each of the three features (steatosis, ballooning, lobular inflammation) was classified as at least grade 1, then the lesion was categorized as NASH. ${ }^{47}$

The SAF (steatosis, activity and fibrosis) score: this score is calculated by the steatosis score (S) that assess the quantities of large or medium-sized lipid droplets, but not foamy microvesicles, from 0 to 3 (S0: $<5 \%$; S1: 5\%-33\%, mild; S2: $34 \%-66 \%$, moderate; S3: $>67 \%$, marked). Activity grade (A, from $0-4$ ) was the unweighted addition of hepatocyte ballooning (0-2) and lobular inflammation $(0-2)$ as described previously. Cases with $\mathrm{A}_{0}(\mathrm{~A}=0)$ had no activity, $A_{1}(A=1)$, mild activity, $A_{2}(A=2)$, moderate activity, $A_{3}(A \geq 3)$ severe activity. Stage of fibrosis $(F)$ was assessed using the score described by NASH-CRN as follows; stage $0\left(\mathrm{~F}_{0}\right)$ none; stage $1\left(\mathrm{~F}_{1}\right)$ : $1 \mathrm{a}$ or $1 \mathrm{~b}$ perisinusoidal zone 3 or $1 \mathrm{c}$ portal fibrosis, stage $2\left(\mathrm{~F}_{2}\right)$ : perisinusoidal and periportal fibrosis without bridging, stage $3\left(\mathrm{~F}_{3}\right)$ : bridging fibrosis and stage $4\left(\mathrm{~F}_{4}\right)$ : cirrhosis. ${ }^{47}$

\section{Complications}

NAFLD, which recently evidenced as a systemic disease, is associated with a significantly higher overall mortality and morbidity compared to the general population, particularly from vascular and liver-related complications. It could be stratified as a major risk factor in developing hypertension, coronary artery diseases, left ventricular diastolic dysfunction and hypertrophy, excessive fat accumulation in the pericardial area, and aortic valve sclerosis, as well as increased carotid artery intimal and medial thickness and prevalence of carotid atherosclerotic plaques. ${ }^{51,52}$ NAFLD, and its associated metabolic factors, also have a negative impact on other liver diseases. With hepatitis $\mathrm{C}$, obesity, IR, and steatosis are predictors of progression to advanced liver fibrosis and are also associated with a lower sustained viral response rates to interferon-based therapies. A proportion of cases of HCC have also been attributed to NAFLD but the overall contribution of metabolic risk factors to HCC is an unresolved issue until now. There is also evidence that the outcome of the alcoholic liver disease is worse in the obese and those with MetS. Fatty liver disease attributable to metabolic risk factors is increasingly noted in those infected with hepatitis B virus (HBV), but whether this has any implications for disease outcomes remains unclear. ${ }^{22}$ In addition, NAFLD could be strongly associated with obstructive sleep apnea syndrome ${ }^{53}$ and chronic kidney diseases. ${ }^{20}$ NAFLD is also associated with colorectal cancer, metabolic bone disease (vitamin D deficiency and osteoporosis) and rare metabolic diseases (lipodystrophies and glycogen storage diseases).$^{54}$ Despite its high prevalence and clinical significance, knowledge and awareness of NAFLD are poor, not only among the general population but also among general practitioners. ${ }^{52}$

\section{Impact of steatosis on LT}

Nowadays, NAFLD has a great concern in regards to LT; from becoming the second most common cause of transplant candidates due to its high worldwide prevalence and from the other hand, the urgent need for increasing the donor pool by using marginal donors with graft steatosis, especially in living donor LT (LDLT). ${ }^{55}$ The assessment of the donor liver fat is a difficult task for the transplant team. An initial evaluation, based on visual inspection and palpation, is first done during graft procurement. However, criteria such as color and texture of the graft depend only on the experience of the explanting surgeon and thus remain subjective. Despite this general agreement, a European survey showed that liver biopsy at the time of procurement for LT is rarely performed. However, several transplant programs consider a liver biopsy as mandatory before discarding a potential liver graft. ${ }^{3}$ It was well recognized that the implantation of a donor's liver with severe fatty infiltration $(>60 \%)$ is frequently associated with an increased incidence of postoperative complications. In the past, grafts with $\geq 30 \%$ fatty infiltration are not acceptable for transplantation..$^{56}$ Furthermore, even the use of donor livers with less fatty infiltration $(<30 \%)$ has been reported to result in a bad outcome after LT. Conversely, some authors reported excellent results after transplantation using severely steatotic liver grafts. ${ }^{57-60}$ Noteworthy, all recipients disclosed a low MELD score and short ischemia times. ${ }^{3}$ However, the critical shortage of organ donors, especially in countries such as middle- and far-eastern countries, where cadaveric donors are almost less available, often mandates the use of such grafts with different degree of steatosis. ${ }^{61}$

It has been postulated that steatotic livers are more sensitive and less tolerant to ischemia-reperfusion (I/R) injury, leading to worse clinical outcomes. If severe, $\mathrm{I} / \mathrm{R}$ injury can lead to liver failure and death. ${ }^{62}$ The exact etiology for the increased susceptibility of steatotic livers to I/R injury is not well-known. Several different hypotheses have been proposed to explain the increased susceptibility, including impaired hepatic microcirculation and mitochondrial dysfunction. ${ }^{62}$ MaS is associated with accumulation of intracellular lipid, increasing hepatocyte volume leading to obstruction of the adjacent sinusoid space, and increasing the vascular resistance in the hepatic microcirculation. ${ }^{63,64}$ This may potentially impair oxygen and nutrient delivery following reperfusion to an already susceptible organ. The increased lipid levels in steatotic livers may also lead to mitochondrial dysfunction through the formation of ROS. ${ }^{65}$ Mitochondrial energy supply is fundamental to cellular viability, and the interruption of key mitochondrial processes disrupts normal cellular bioenergetics, 
impairs cellular function, and subsequently leads to cell death by either necrosis or apoptosis. ${ }^{66}$ However, MiS is frequently regarded as a mild and reversible condition and is usually disregarded as a risk factor for graft dysfunction, it has been established that graft MiS is an independent factor influencing the early graft function (EGD). ${ }^{61}$ Cieslak et al. ${ }^{67}$ observed that a $12 \%$ increase in MiS was equivalent to a $50 \%$ increased risk for graft dysfunction. ${ }^{67} \mathrm{MiS}$ may develop because of severe and prolonged inhibition of mitochondrial $\beta$-oxidation of FAs during preservation-reperfusion injury. ${ }^{68} \mathrm{MiS}$ increases progressively and evolves in the liver during cold storage and worsens significantly before reperfusion in the recipient, whereas the degree of MaS remains constant. ${ }^{69}$ On the contrary and despite the graft dysfunction risk, the use of livers exhibiting MiS does not decrease graft or patient survival rates. ${ }^{61}$

On the other hand, the composition of hepatic lipids and particularly thromboxane $\mathrm{A}_{2}$ levels are considered the principal modulators of $\mathrm{I} / \mathrm{R}$ injury in the steatotic livers, while the size and morphology of lipid droplets are insignificant. ${ }^{70}$ Other potential mechanisms that have been proposed include Kupffer cell dysfunction, ${ }^{71}$ more lipid peroxidation, ${ }^{72,73}$ more exuberant pro-inflammatory responses, including increased release of pro-inflammatory mediators such as TNF- $\alpha,{ }^{74}$ and impaired leukocyte adhesion. ${ }^{75}$ Therefore, it is likely that the increased vulnerability of the steatotic livers to more $I / R$ injury are multifactorial, and further experimental studies are required to elucidate the underlying mechanisms. ${ }^{62}$ Various types of I/R injury are implemented during both the donors and recipients' hepatic surgery, including warm ischemia in hepatic resection when hepatic inflow is temporarily occluded and cold and re- warming I/R injury when a donor's liver is re-perfused during LT. ${ }^{76}$

\section{Warm Ischemia Time (WIT)}

Whereas sinusoidal endothelial cells are the cells in the liver most susceptible to cold ischemia, hepatocytes are injured more readily by warm ischemia. ${ }^{69}$ Experimentally, a WIT of 30 minutes plus 4-5hours of cold preservation causes primary non-function (PNF) and the longer the WIT, the greater the cumulative release of ALT, AST, and lactate dehydrogenase in the perfusion solution. On the other hand, a shorter WIT increases the graft tolerance to cold storage with better outcomes. ${ }^{77}$ The longer the WIT results in Hepatocyte vacuolation, sinusoidal congestion, and the appearance and aggravation of focal hepatocyte drop-out. ${ }^{78}$ Simultaneously, the recipient will suffer from acute hemodynamic changes and an eliminated hepatic metabolism, as well as the cytokine, metabolite, and other toxin accumulation in the splanchnic system. ${ }^{79}$

\section{Cold Ischemia Time (CIT)}

This is defined as the time from cardiac arrest or cross-clamping and the initiation of in-situ cold flush in the donor to the removal of the organ from cold storage for implantation into the recipient. ${ }^{80}$ Cold ischemia or cold storage is another factor that interferes with the incidence of graft dysfunction. The cutoff for CIT is not uniform; some studies reported that a CIT $>10$ hours is associated with poor outcomes after LT, ${ }^{81,82}$ and others have reported that CIT can be longer than 18 hours. ${ }^{48,83}$ Decreasing temperatures can induce cell injury through the release of proteases, leading to alterations in calcium homeostasis, modifications of the cytoskeleton, and local tissue destruction. ${ }^{84}$ Cold preservation also induces several changes in the hepatocytes, such as cell swelling, intracellular acidosis, loss of mitochondrial function, and decreased ATP. ${ }^{76}$ An analysis of donor and transplant- related factors using united network of organ sharing (UNOS)/organ procurement and transplantation network registry data has shown that for every hour of CIT above 8hours, the adjusted risk of graft failure increases by $2 \%$. This has been confirmed in the continental European liver transplant experience; every 15 minute increase in CIT increases 1year graft failure risk by $0.9 \%$. In addition, the risk of developing non-anastomotic biliary strictures significantly increased with every hour increase in CIT, as biliary epithelium may be particularly sensitive to ischemia-induced oxidative stress after reperfusion. ${ }^{80}$ In a recent systematic review on experimental studies, increased ischemia times, as well as increased severity of steatosis, was shown to correlate with a decrease in survival. Histologically, $\geq 30 \% \mathrm{MaS}$ was associated with increased intraparenchymal hemorrhage, sinusoidal congestion, and necrosis. ALT, AST, prothrombin time, and bilirubin were increased in subjects with $\geq 30 \% \mathrm{MaS}$ or mixed hepatic steatosis. Similar degrees of histological injury and levels of transaminases in MiS were noticed. ${ }^{62}$

Moreover, almost all LT recipients are maintained on calcineurin inhibitors, which are associated with increased generation of ROS, more mitochondrial dysfunction, and lipid peroxidation. This may have important implications for the natural history of recurrence of NAFLD following LT. Whether calcineurin inhibitor dosing should be minimized in patients with recurrence of NASH is not known, but a theoretical possibility for such an approach to recurrence of NASH following LT exists. ${ }^{85}$ The incidence of PNF after LT is $1.8 \%$ in normal grafts and $5.1 \%$ in those exhibiting greater than $30 \%$ steatosis. Therefore, steatosis $\geq 30 \%$ in a donor's liver is considered unacceptable. However, other studies demonstrated that moderate to severe steatosis is acceptable when other risk factors are excluded. ${ }^{76}$ A large study using UNOS data showed that the presence of $\geq 30 \%$ of $\mathrm{MaS}$ was an independent risk factor for impaired 1-year graft survival. ${ }^{86}$ Interestingly, two Italian groups demonstrated that transplanting livers with moderate to severe MaS is an independent risk factor for the development of biliary complications after LT. ${ }^{87,88}$ Perhaps, an impaired microcirculation at the sinusoidal level could be responsible for bile duct ischemic damage, resulting in a higher risk of biliary strictures. ${ }^{3}$

\section{Treatment}

In spite of the growing understanding of the global epidemic of NAFLD, there is no definite pharmacotherapy available. Only proposed benefits according to available evidence as lifestyle modification with regular exercise and dietary changes or bariatric surgery in morbidly obese patients are available. Other suggested means of pharmacological therapy as anti-inflammatory, insulin sensitizers or antioxidant agents have shown promising results, there is some concern for adverse effects and long-term safety. ${ }^{89}$

Long-term strategies: These strategies are applicable for the treatment of hepatic steatosis in general population aiming for decreasing the impact of NAFLD on the general health. In addition, sticking to the appropriate strategies can increase the donor pool through providing healthier persons with healthier liver grafts. They are of greater concerns in LDLT programs.

Non-pharmacologic treatment (lifestyle modification): These interventions, aimed at correcting unhealthy lifestyles, simultaneously treat all the clinical manifestations of the MetS and are an effective treatment option in NAFLD. Lifestyle changes include weight loss, dietary changes, reduction of sedentarily and physical exercise. They 
are best accomplished through cognitive-behavioral therapy, which should always be implemented as a first-line therapeutic option, regardless of the severity NAFLD/NASH. ${ }^{11}$ European association for the study of the liver (EASL), diabetes (EASD), and obesity (EASO) clinical practice guidelines for the management of NAFLD proposed pragmatic approaches for lifestyle modification, ${ }^{54}$ including:

Energy restriction, in the form of 500-1000kcal energy defect, to induce a weight loss of $500-1000 \mathrm{~g} /$ week with $7-10 \%$ total weight loss target in obese patients. In addition, Long-term maintenance approach, combining physical activity according to the principles of cognitive-behavioral treatment should be achieved.

Dietary modification with adherence to the Mediterranean diet has been reported to reduce liver fat, including low-to-moderate fat and moderate-to-high carbohydrate intake, low-carbohydrate ketogenic diet or high-protein diets, and avoid fructose-containing beverages and foods. ${ }^{90,91}$ Saturated fatty acids should be limited while increased consumption of food rich in mono- and polyunsaturated fatty acids (Omega-3) is advised. ${ }^{92}$

Exercise/physical activity; 150-200min/week of moderateintensity aerobic physical activities in $3-5$ sessions are generally preferred (brisk walking or stationary cycling). Resistance training is also effective and promotes musculoskeletal fitness, with effects on metabolic risk factors. Avoiding of a high rate of inactivity-promoting fatigue and daytime sleepiness as they reduce compliance with exercises.

Pharmacologic treatment: Drug therapy should be indicated for progressive NASH (bridging fibrosis and cirrhosis) but also for earlystage NASH with increased risk of fibrosis progression (age $>50$ years, diabetes, MetS, increased ALT) or active NASH with high necroinflammatory activity. Whereas no drug has currently been tested in phase III trials and is approved for NASH by regulatory agencies, no specific therapy can be firmly recommended. Safety and tolerability are essential prerequisites for drug treatment, because of NASHassociated co-morbidities and polypharmacy, a potential source of drug-drug interactions. ${ }^{54}$

Insulin sensitizers: Regarding metformin, there are scarce evidences for its histological efficacy in NASH. Thiazolidinediones have insulin-sensitizing effects. The pioglitazone vs. vitamin E vs. placebo for non-alcoholic steatohepatitis (PIVENS) trial for 2years in patients without overt diabetes demonstrated that Pioglitazone improved all histological features (except for fibrosis) and achieved resolution of NASH more often than placebo. ${ }^{93}$ These histological benefits occurred together with ALT improvement and partial correction of IR. Despite the safety and tolerability profile, pioglitazone can be used for selected patients with NASH, particularly in T2DM where the drug has a registered use and caution should be taken in regard to its cardiovascular risks. ${ }^{54}$

Anti-obesity and lipid lowering agents: Drug therapies of obesity (orlistat and sibutramine) have not shown a direct beneficial effect on the liver independent of the beneficial effect of the weight loss. However, these drugs may enhance the beneficial effects of behavioral therapy ${ }^{11}$ Lipid lowering agents (statins, fibrates, and omega-3 FAs) use in NAFLD are safe, with no increased risk of hepatotoxicity, and may even reduce aminotransferases with a possible role in delaying progression from NAFL to NASH. ${ }^{54}$

Antioxidant and cytoprotective agents: in the PIVENS trial, vitamin E (800IU/day) improved steatosis, inflammation and ballooning and induced resolution of NASH in 36\% of patients vs. $21 \%$ in the placebo arm..$^{93}$ In addition; vitamin E may have a greater benefit when combined with other agents as ursodeoxycholic acid. Contrarily, concerns about vitamin E long-term safety exist as it may be associated with increased risk for cardiovascular events and prostate cancer in older patients $>50$ years. ${ }^{89}$ Obeticholic acid, which is a synthetic farnesoid X receptor agonist, decreased IR in T2DM and improved all NASH lesions and fibrosis in non-cirrhotic NASH patients after 72 weeks of treatment. ${ }^{94}$

Other Trials: Promising novel agents with anti-inflammatory (Pentoxifylline) and antifibrotic agents that may improve liver enzymes and histology are also being tested in late-phase RCTs in NASH. ${ }^{54}$ Coffee is rich in sources of bioactive phytochemicals including methylxanthines (caffeine), amino acids, phenolic acids, and polyphenols which may protect against liver disease. ${ }^{95}$ In a recent meta-analysis of 16 studies comprising 3153 cases of hepatocellular carcinoma, the risk of HCC was found to be reduced by $40 \%$ with coffee intake compared to no coffee. ${ }^{96}$ Emerging evidence, in animal studies, has shown that inhibiting renin angiotensin aldosterone system pathway decreases hepatic stellate cell activity, which in turn prevents fibrosis. Therefore, angiotensin converting enzyme (ACE) inhibitors and angiotensin receptor blockers may be useful in treating NAFL/NASH as they may lead to decreased fibrosis. However, not many studies have been done in humans with NAFLD to assess the effectiveness of ACE inhibitors or angiotensin receptor blockers. ${ }^{89}$

\section{Surgical treatment}

Venesection: Hepatic iron accumulation is associated with IR, and iron depletion improves IR. In NAFLD, high ferritin levels are common. In these patients, a phlebotomy program to reduce iron stores to near iron deficiency improved the NAS, without worsening the fibrosis, but more data are needed. ${ }^{54}$

Bariatric surgery: Although bariatric surgery is not specifically indicated in NAFLD, it may be useful in morbidly obese patients. A systematic review with meta-analysis has reported an improved liver histology following gastric bypass and laparoscopic banding. ${ }^{97}$ Moreover, a recent cohort study confirmed that bariatric surgeryassociated weight loss cleared NASH in $85 \%$ of patients and improved fibrosis in $34 \%$, although the possible benefits should be balanced against perioperative complications. ${ }^{98}$

Short-term strategies: These strategies are reserved to the short perioperative period aiming for improving the outcomes after LT of a steatotic liver graft. They could be also useful in deceased donors. These strategies include ischemic preconditioning (IP) and liver defatting approaches.

Ischemic preconditioning: Several techniques have been developed as IP to improve the detrimental effects of $I / R$ injury on marginal donor grafts, in particular, steatotic ones. Clinical studies have shown decreasing hepatocyte swelling and enzyme release after LT as well as less autophagy and possibly less rejection in steatotic livers exposed to IP. ${ }^{99,100}$ A recent systematic review of experimental IP in steatotic livers ${ }^{101}$ concluded that animals with preconditioned livers had improved outcomes, including increased survival, less histologic injury and improved liver function. ${ }^{102}$ Several approaches for the IP were examined experimentally, including:

Pharmacological preconditioning: such as with anaesthetics, alphaand beta-adrenergic blockers, antioxidants, and/or steroids have been suggested as potential hepatoprotective strategies for steatotic livers, 
but few have been reported in humans. Experimentally, some of these agents have been added to the cold storage preservation solution and found to reduce I/R injury-related markers in perfused steatotic livers. Beck-Schimmer et al. ${ }^{103}$ hypothesized; in an RCT that sevoflurane anaesthesia would reduce postoperative liver injury and promote beneficial effects from an inducible nitric oxide pathway. In addition, it can significantly reduce the postoperative aminotransferase levels, in particularly in the steatotic livers. ${ }^{103}$ Carvedilol, a beta- and alphaadrenergic blocker to treat ischemic heart, was found also to reduce hepatic death markers, vascular resistance and ROS, as well as increased bile production and hepatic ATP levels post-reperfusion. ${ }^{104}$ Moreover, intravenously administered the antioxidant glutathione or its precursor $\mathrm{N}$-acetylcysteine shortly prior to reperfusion recovered the normal intracellular glutathione level and significantly reduced lipid peroxidation, I/R injury markers and hepatic death. ${ }^{73,105}$ It is worth noting that none of these studies investigated the possibility of combining several pharmacological agents at once; therefore, it is possible that doing so would lead to a more dramatic decrease in sensitivity to I/R injury. ${ }^{102,106}$

Surgical preconditioning: in which, the major blood vessels to the liver are clamped momentarily (Pringle manoeuvre). This has been shown to reduce lipid peroxidation, hepatic microcirculation failure, and neutrophil accumulation after subsequent I/R injury when applied to steatotic livers. IP has been shown to protect human livers against a subsequent period of ischemia in patients undergoing hemi hepatectomy as in live donors. The analysis of a subgroup of patients with mild-to-moderate steatosis presented reduced serum levels of liver damage markers (amino transferases) when preconditioned. ${ }^{107}$

Heat shock preconditioning: Another experimental approach to alleviate I/R injury is heat shock preconditioning, in which fatty liver donor rats were exposed to brief whole-body hyperthermia (10minutes at 42.5 degrees Celsius) and allowed to recover. ${ }^{108}$ This approach has been shown to preserve microcirculatory parameters (sinusoidal perfusion rate, sinusoidal diameter, minimal leukocyte-endothelial adhesion) and prevent microvascular perfusion failure after surgically induced I/R in obese rats with steatotic livers. ${ }^{109}$ Interestingly, there was a time window of efficacy ranging from $6 \mathrm{~h}$ to $24 \mathrm{~h}$ after the heat shock. The protective mechanisms of heat shock preconditioning are not fully understood, but the utility of this approach warrants further studies that would enable a rational design of heat shock preconditioning regimens to improve effectiveness and practicality. ${ }^{106}$

Liver defatting: Multiple studies described what is called "liver defatting", which have been performed in attempts to optimize the steatotic liver grafts prior to LT. ${ }^{106,110}$

Combined measures (Dietary, exercise and drug therapy): A short-term intensive treatment with protein-rich diet (1000kcal/day), exercise (600 kcal/day), and bezafibrate $(400 \mathrm{mg} /$ day) for $2-8$ weeks in a small trial of 11donors led to threefold decrease in MaS and reduced body weight and BMI. Alternatively, the consumption of omega-3 FAs for 1 month was used to decrease MaS in three candidates for LDLT who had biopsy-proven hepatic MaS $>30 \%$ prior to donation. ${ }^{110}$ In addition, administration of omega- 3 FAs enhances the hepatic regeneration and functional recovery in the living donors. ${ }^{111}$

Machine perfusion: The use of machine-based liver perfusion systems may also offer benefits and perhaps a way to test the function of the organ prior to implantation. The new preservation concepts include in situ warm oxygenated perfusion before the organ harvest (normothermic concept) ${ }^{112}$ or hypothermic machine perfusion after organ procurement and transport to the transplantation centre (hypothermic concept). ${ }^{113}$ While the perfusion system may enable to determine the potential viability of the graft, wide application of perfusion system in marginal graft such as severe steatotic livers will need long-term data after LT. ${ }^{3}$

Optimizing the perfusion solution: ultimately, the goal of liver defatting is to rapidly decrease the proportion of MaS hepatocytes, while maintaining high viability and functionality. Steatosis is the result of an imbalance between triglyceride synthesis and breakdown processes in hepatocytes. Therefore, to achieve significant defatting, the protocol of choice should shift this balance towards more efficient breakdown (lipolysis) and excretion of related by-products, as well as minimizing the synthesis. ${ }^{114,115}$ The pathways responsible for lipid metabolism are well known; however, there is much work to be done on how best to modulate this metabolism using cocktails of agents in order to achieve rapid defatting without adversely affecting viability and other critical liver functions. As illustrated in figure 13 , targeting multiple routes simultaneously may provide the most effective approach, but the rational design of defatting protocols will require a better understanding of the potential interactions among the relevant metabolic pathways in steatotic hepatocytes. This would be greatly facilitated if a suitable cell culture model of hepatic steatosis were available to perform both screening and mechanistic studies in an efficient manner, prior to testing in actual livers. ${ }^{106}$ While many challenges need to be overcome, liver defatting is a potentially promising approach to reduce the sensitivity of steatotic livers to $\mathrm{I} / \mathrm{R}$ injury, and a new modality that may enable the successful recovery of a large number of livers that would otherwise be discarded. ${ }^{106}$

\section{Conclusion}

Using the marginal donors especially those with NAFLD will markedly increase the donor pool for LT. Consequently, it will shorten the recipient deaths on the lengthy waiting list for LT. Several approaches, including long-term and short-term measures were identified for the management of NAFLD.

\section{Acknowledgement}

The authors would like to thank Dr. Jennifer Gomez for her kind invitation.

\section{Conflict of interest}

The authors declare that there is no conflict of interests regarding the publication of this paper.

\section{References}

1. Makowka L, Gordon RD, Todo S, et al. Analysis of donor criteria for the prediction of outcome in clinical liver transplantation. Transplant Proc. 1987;19(1 Pt 3):2378-2382.

2. Attia M, Silva MA, Mirza DF. The marginal liver donor-an update. Transpl Int. 2008;21(8):713-724.

3. McCormack L, Dutkowski P, El-Badry AM, et al. Liver transplantation using fatty livers: always feasible? J Hepatol. 2011;54(5):1055-1062.

4. Käräjämäki AJ, Patsi OP, Savolainen M, et al. Non-Alcoholic Fatty Liver Disease as a Predictor of Atrial Fibrillation in Middle-Aged Population (OPERA Study). PLoS One. 2015;10(11):e0142937.

5. Sanyal AJ. AGA technical review on nonalcoholic fatty liver disease. Gastroenterology. 2002;123(5):1705-1725. 
6. Chalasani N, Younossi Z, Lavine JE, et al. The diagnosis and management of non-alcoholic fatty liver disease: Practice guideline by the American Association for the Study of Liver Diseases, American College of Gastroenterology, and the American Gastroenterological Association. Hepatology. 2012;55(6):2005-2023.

7. Nocito A, El-Badry AM, Clavien PA. When is steatosis too much for transplantation? J Hepatol. 2006;45(4):494-499.

8. Wilkins T, Tadkod A, Hepburn I, et al. Nonalcoholic fatty liver disease: diagnosis and management. Am Fam Physician. 2013;88(1):35-42.

9. Bhala N, Jouness RI, Bugianesi E. Epidemiology and natural history of patients with NAFLD. Curr Pharm Des. 2013;19(29):5169-5176.

10. Younossi ZM, Koenig AB, Abdelatif D, et al. Global Epidemiology of Non-Alcoholic Fatty Liver Disease-Meta-Analytic Assessment of Prevalence, Incidence and Outcomes. Hepatology. 2015;64(1):73-84.

11. Loria P, Adinolfi LE, Bellentani S, et al. Practice guidelines for the diagnosis and management of nonalcoholic fatty liver disease. A decalogue from the Italian Association for the Study of the Liver (AISF) Expert Committee. Dig Liver Dis. 2010;42(4):272-282.

12. Bugianesi E, Gastaldelli A, Vanni E, et al. Insulin resistance in nondiabetic patients with non-alcoholic fatty liver disease: sites and mechanisms. Diabetologia. 2005;48(4):634-642.

13. Donnelly KL, Smith CI, Schwarzenberg SJ, et al. Sources of fatty acids stored in liver and secreted via lipoproteins in patients with nonalcoholic fatty liver disease. J Clin Invest. 2005;115(5):1343-1351.

14. Tolman KG, Dalpiaz AS. Treatment of non-alcoholic fatty liver disease. Ther Clin Risk Manag. 2007;3(6):1153-1163.

15. Cusi K. Role of obesity and lipotoxicity in the development of nonalcoholic steatohepatitis: pathophysiology and clinical implications. Gastroenterology. 2012;142(4):711-725.

16. Reid AE. Nonalcoholic steatohepatitis. Gastroenterology. 2001;121(3):710-723.

17. Pessayre D, Berson A, Fromenty B, et al. Mitochondria in steatohepatitis. Semin Liver Dis. 2001;21(1):57-69.

18. Duseja A, Chawla YK. Obesity and NAFLD: the role of bacteria and microbiota. Clin Liver Dis. 2014;18(1):59-71.

19. Argo CK, Northup PG, Al-Osaimi AM, et al. Systematic review of risk factors for fibrosis progression in non-alcoholic steatohepatitis. $J$ Hepatol. 2009;51(2):371-379.

20. Fotbolcu H, Zorlu E. Nonalcoholic fatty liver disease as a multi-systemic disease. World J Gastroenterol. 2016;22(16):4079-4090.

21. Sass DA, Chang P, Chopra KB. Nonalcoholic fatty liver disease: a clinical review. Dig Dis Sci. 2005;50(1):171-180.

22. Chitturi S, Farrell GC, Hashimoto E, et al. Non-alcoholic fatty liver disease in the Asia-Pacific region: definitions and overview of proposed guidelines. J Gastroenterol Hepatol. 2007;22(6):778-787.

23. Schwenger KJ, Allard JP. Clinical approaches to non-alcoholic fatty liver disease. World J Gastroenterol. 2014;20(7):1712-1723.

24. Bedogni G, Bellentani S, Miglioli L, et al. The Fatty Liver Index: a simple and accurate predictor of hepatic steatosis in the general population. BMC Gastroenterol. 2006;6:33.

25. Dixon JB, Bhathal PS, O'Brien PE. Nonalcoholic fatty liver disease: predictors of nonalcoholic steatohepatitis and liver fibrosis in the severely obese. Gastroenterology. 2001;121(1):91-100.

26. Campos GM, Bambha K, Vittinghoff E, et al. A clinical scoring system for predicting nonalcoholic steatohepatitis in morbidly obese patients. Hepatology. 2008;47(6):1916-1923.
27. Zein, Edmilson, Schluchter, et al. NASH predictive index (NPI) for use in patients with nonalcoholic fatty liver disease. Hepatology. 2007:46.

28. Poynard T, Ratziu V, Charlotte F, et al. Diagnostic value of biochemica markers (Nash Test) for the prediction of non alcoholo steato hepatitis in patients with non-alcoholic fatty liver disease. BMC Gastroenterol. 2006;6:34.

29. McPherson S, Stewart SF, Henderson E, et al. Simple non-invasive fibrosis scoring systems can reliably exclude advanced fibrosis in patients with non-alcoholic fatty liver disease. Gut. 2010;59(9):1265-1269.

30. Tapper EB, Krajewski K, Lai M, et al. Simple non-invasive biomarkers of advanced fibrosis in the evaluation of non-alcoholic fatty liver disease. Gastroenterol Rep (Oxf). 2014;2(4):276-280.

31. Angulo P, Hui JM, Marchesini G, et al. The NAFLD fibrosis score: a noninvasive system that identifies liver fibrosis in patients with NAFLD 2007;45(4):846-854.

32. Alkhouri N, Feldstein AE. Noninvasive diagnosis of nonalcoholic fatty liver disease: Are we there yet? Metabolism. 2016;65(8):1087-95.

33. Wu SD, Wang JY, Li L. Staging of liver fibrosis in chronic hepatitis B patients with a composite predictive model: a comparative study. World J Gastroenterol. 2010;16(4):501-507.

34. Calès P, Laine F, Boursier J, et al. Comparison of blood tests for liver fibrosis specific or not to NAFLD. J Hepatol. 2009;50(1):165-173.

35. Harrison SA, Oliver D, Arnold HL, et al. Development and validation of a simple NAFLD clinical scoring system for identifying patients without advanced disease. Gut. 2008;57(10):1441-1447.

36. Ratziu V, Giral P, Charlotte F, et al. Liver fibrosis in overweight patients. Gastroenterology. 2000;118(6):1117-1123.

37. Shah AG, Lydecker A, Murray K, et al. Comparison of noninvasive markers of fibrosis in patients with nonalcoholic fatty liver disease. Clin Gastroenterol Hepatol. 2009;7(10):1104-1112.

38. Ratziu V, Massard J, Charlotte F, et al. Diagnostic value of biochemical markers (FibroTest-FibroSURE) for the prediction of liver fibrosis in patients with non-alcoholic fatty liver disease. BMC Gastroenterol. 2006;6:6.

39. Guha IN, Parkes J, Roderick P, et al. Noninvasive markers of fibrosis in nonalcoholic fatty liver disease: Validating the European Liver Fibrosis Panel and exploring simple markers. Hepatology. 2008;47(2):455-460.

40. Palekar NA, Naus R, Larson SP, et al. Clinical model for distinguishing nonalcoholic steatohepatitis from simple steatosis in patients with nonalcoholic fatty liver disease. Liver Int. 2006;26(2):151-156.

41. Joseph AE, Saverymuttu SH, Al-Sam S, et al. Comparison of liver histology with ultrasonography in assessing diffuse parenchymal liver disease. Clin Radiol. 1991;43(1):26-31.

42. Neuschwander-Tetri BA, Caldwell SH. Nonalcoholic steatohepatitis: summary of an AASLD Single Topic Conference. Hepatology. 2003;37(5):1202-1219.

43. Mazhar SM, Shiehmorteza M, Sirlin CB. Noninvasive assessment of hepatic steatosis. Clin Gastroenterol Hepatol. 2009;7(2):135-140.

44. Festi D, Schiumerini R, Marzi L, et al. Review article: the diagnosis of non-alcoholic fatty liver disease-availability and accuracy of noninvasive methods. Aliment Pharmacol Ther. 2013;37(4):392-400.

45. Van Werven JR, Marsman HA, Nederveen AJ, et al. Assessment of hepatic steatosis in patients undergoing liver resection: comparison of US, CT, T1-weighted dual-echo MR imaging, and point-resolved $1 \mathrm{H} \mathrm{MR}$ spectroscopy. Radiology. 2010;256(1):159-168.

46. Fazel Y, Koenig AB, Sayiner M, et al. Epidemiology and natural history of non-alcoholic fatty liver disease. Metabolism. 2016;65(8):1017-1025. 
47. Bedossa P, Poitou C, Veyrie N, et al. Histopathological algorithm and scoring system for evaluation of liver lesions in morbidly obese patients. Hepatology. 2012;56(5):1751-1759.

48. Ploeg RJ, D’Alessandro AM, Knechtle SJ, et al. Risk factors for primary dysfunction after liver transplantation-a multivariate analysis Transplantation. 1993;55(4):807-813.

49. Brunt EM, Kleiner DE, Wilson LA, et al. Nonalcoholic fatty liver disease (NAFLD) activity score and the histopathologic diagnosis in NAFLD: distinct clinicopathologic meanings. Hepatology. 2011;53(3):810-820.

50. Kleiner DE, Brunt EM, Van Natta M, et al. Design and validation of a histological scoring system for nonalcoholic fatty liver disease. Hepatology. 2005;41(6):1313-1321.

51. Lonardo A, Ballestri S, Marchesini G, et al. Nonalcoholic fatty liver disease: a precursor of the metabolic syndrome. Dig Liver Dis. 2015;47(3):181-190.

52. Nascimbeni F, Pais R, Bellentani S, et al. From NAFLD in clinical practice to answers from guidelines. J Hepatol. 2013;59(4):859-871.

53. Sookoian S, Pirola CJ. Obstructive sleep apnea is associated with fatty liver and abnormal liver enzymes: a meta-analysis. Obes Surg. 2013;23(11):1815-1825

54. EASL-EASD-EASO Clinical Practice Guidelines for the management of non-alcoholic fatty liver disease. J Hepatol. 2016;64(6):1388-1402.

55. Zezos P, Renner EL. Liver transplantation and non-alcoholic fatty liver disease. World J Gastroenterol. 2014;20(42):15532-15538.

56. Todo S, Demetris AJ, Makowka L, et al. Primary nonfunction of hepatic allografts with preexisting fatty infiltration. Transplantation. 1989;47(5):903-905

57. McCormack L, Petrowsky H, Jochum W, et al. Use of severely steatotic grafts in liver transplantation: a matched case-control study. Ann Surg. 2007;246(6):940-948.

58. Noujaim HM, de Ville de Goyet J, Montero EF, et al. Expanding postmortem donor pool using steatotic liver grafts: a new look. Transplantation. 2009;87(6):919-925.

59. Wong TC, Fung JY, Chok KS, et al. Excellent outcomes of liver transplantation using severely steatotic grafts from brain-dead donors Liver Transpl. 2016;22(2):226-236.

60. Tandoi F, Salizzoni M, Brunati A, et al. Excellent outcomes of liver transplantation using severely steatotic grafts from brain-dead donors Liver Transpl. 2016;22(3):377-378.

61. Soejima Y, Shimada M, Suehiro T, et al. Use of steatotic graft in livingdonor liver transplantation. Transplantation. 2003;76(2):344-348.

62. Chu MJ, Hickey AJ, Phillips AR, et al. The impact of hepatic steatosis on hepatic ischemia-reperfusion injury in experimental studies: a systematic review. Biomed Res Int. 2013;2013:192029.

63. Ijaz S, Yang W, Winslet MC, et al. Impairment of hepatic microcirculation in fatty liver. Microcirculation. 2003;10(6):447-456.

64. Farrell GC, Teoh NC, McCuskey RS. Hepatic microcirculation in fatty liver disease. Anat Rec (Hoboken). 2008;291(6):684-692.

65. Trauner M, Arrese M, Wagner M. Fatty liver and lipotoxicity. Biochim Biophys Acta. 2010;1801(3):299-310.

66. Hand SC, Menze MA (2008) Mitochondria in energy-limited states: mechanisms that blunt the signaling of cell death. J Exp Biol. 2008;211(P 12):1829-1840.

67. Cieślak B, Lewandowski Z, Urban M, et al. Microvesicular liver graft steatosis as a risk factor of initial poor function in relation to suboptimal donor parameters. Transplant Proc. 2009;41(8):2985-2988.
68. Lawal A, Florman S, Fiel MI, et al. Identification of ultrastructural changes in liver allografts of patients experiencing primary nonfunction. Transplant Proc. 2005;37(10):4339-4342.

69. Silva MA, Mirza DF, Murphy $\mathrm{N}$, et al. Intrahepatic complemen activation, sinusoidal endothelial injury, and lactic acidosis are associated with initial poor function of the liver after transplantation. Transplantation. 2008;85(5):718-725.

70. El-Badry AM, Jang JH, Elsherbiny A, et al. Chemical composition of hepatic lipids mediates reperfusion injury of the macrosteatotic mouse liver through thromboxane A(2). J Hepatol. 2011;55(6):1291-1299.

71. Zhong Z, Connor H, Stachlewitz RF, et al. Role of free radicals in primary nonfunction of marginal fatty grafts from rats treated acutely with ethanol. Mol Pharmacol. 1997;52(5):912-919.

72. Berthiaume F, Barbe L, Mokuno Y, et al. Steatosis reversibly increases hepatocyte sensitivity to hypoxia-reoxygenation injury. J Surg Res. 2009;152(1):54-60.

73. Serafín A, Roselló-Catafau J, Prats N, et al. Ischemic preconditioning increases the tolerance of Fatty liver to hepatic ischemia-reperfusion injury in the rat. Am J Pathol. 2002;161(2):587-601.

74. Vairetti M, Ferrigno A, Carlucci F, et al. Subnormothermic machine perfusion protects steatotic livers against preservation injury: a potential for donor pool increase?. Liver Transpl. 2009;15(1):20-29.

75. Teramoto K, Bowers JL, Kruskal JB, et al. Hepatic microcirculatory changes after reperfusion in fatty and normal liver transplantation in the rat. Transplantation. 1993;56(5):1076-1082.

76. Chen XB, Xu MQ. Primary graft dysfunction after liver transplantation Hepatobiliary Pancreat Dis Int. 2014;13(2):125-137.

77. Obara H, Matsuno N, Enosawa S, et al. Pretransplant screening and evaluation of liver graft viability using machine perfusion preservation in porcine transplantation. Transplant Proc. 2012;44(4):959-961.

78. Monbaliu D, Libbrecht L, De Vos R, et al. The extent of vacuolation in non-heart-beating porcine donor liver grafts prior to transplantation predicts their viability. Liver Transpl. 2008;14(9):1256-1265.

79. Ijtsma AJ, van der Hilst CS, De Boer MT, et al. The clinical relevance of the anhepatic phase during liver transplantation. Liver Transpl. 2009; 15(9):1050-1055.

80. Feng S, Lai JC. Expanded criteria donors. Clin Liver Dis. 2014;18(3):633-649.

81. Johnson SR, Alexopoulos S, Curry M, et al. Primary nonfunction (PNF) in the MELD Era: An SRTR database analysis. Am J Transplant. 2007;7(4):1003-1009.

82. Cameron AM, Ghobrial RM, Yersiz H, et al. Optimal utilization of donor grafts with extended criteria: a single-center experience in over 1000 liver transplants. Ann Surg. 2006;243(6):748-753.

83. Brokelman W, Stel AL, Ploeg RJ. Risk factors for primary dysfunction after liver transplantation in the University of Wisconsin solution era Transplant Proc. 1999;31(5):2087-2090.

84. Defamie V, Cursio R, Le Brigand K, et al. Gene expression profiling of human liver transplants identifies an early transcriptional signature associated with initial poor graft function. Am $J$ Transplant. 2008;8(6):1221-1236

85. Busuttil RW, Klintmalm GB. Transplantation of the liver. 3rd ed. Philadelphia, USA: Elsevier Saunders; 2015.

86. Spitzer AL, Lao OB, Dick AA, et al. The biopsied donor liver: incorporating macrosteatosis into high-risk donor assessment. Liver Transpl. 2010;16(7):874-884. 
87. Baccarani U, Isola M, Adani GL, et al. Steatosis of the hepatic graft as a risk factor for post-transplant biliary complications. Clin Transplant. 2010;24(5):631-635.

88. Frongillo F, Lirosi MC, Sganga G, Grossi U, Nure E, et al. (2014) Graft steatosis as a risk factor of ischemic-type biliary lesions in liver transplantation. Transplant Proc. 2014;46(7):2293-2294.

89. Hossain N, Kanwar P, Mohanty SR. A Comprehensive Updated Review of Pharmaceutical and Nonpharmaceutical Treatment for NAFLD. Gastroenterol Res Pract. 2016;2016:7109270.

90. Ryan MC, Itsiopoulos C, Thodis T, et al. The Mediterranean diet improves hepatic steatosis and insulin sensitivity in individuals with nonalcoholic fatty liver disease. J Hepatol. 2013;59(1):138-143.

91. Thoma C, Day CP, Trenell MI. Lifestyle interventions for the treatment of non-alcoholic fatty liver disease in adults: a systematic review. $J$ Hepatol. 2012;56(1):255-266.

92. Chalasani N, Younossi Z, Lavine JE, et al. The diagnosis and management of non-alcoholic fatty liver disease: practice guideline by the American Gastroenterological Association, American Association for the Study of Liver Diseases, and American College of Gastroenterology. Gastroenterology. 2012;142(7):1592-1609.

93. Sanyal AJ, Chalasani N, Kowdley KV, et al. Pioglitazone, vitamin E, or placebo for nonalcoholic steatohepatitis. $N$ Engl J Med. 2010;362(18):1675-1685.

94. Mudaliar S, Henry RR, Sanyal AJ, et al. Efficacy and safety of the farnesoid $\mathrm{X}$ receptor agonist obeticholic acid in patients with type 2 diabetes and nonalcoholic fatty liver disease. Gastroenterology. 2013;145(3):574.e.1-582.e1

95. Anty R, Marjoux S, Iannelli A, et al. Regular coffee but not espresso drinking is protective against fibrosis in a cohort mainly composed of morbidly obese European women with NAFLD undergoing bariatric surgery. J Hepatol. 2012;57(5):1090-1096.

96. Bravi F, Bosetti C, Tavani A, et al. Coffee reduces risk for hepatocellular carcinoma: an updated meta-analysis. Clin Gastroenterol Hepatol. 2013;11(11):1413.e.1-1421.e1.

97. Mummadi RR, Kasturi KS, Chennareddygari S, et al. Effect of bariatric surgery on nonalcoholic fatty liver disease: systematic review and metaanalysis. Clin Gastroenterol Hepatol. 2008;6(12):1396-1402.

98. Lassailly G, Caiazzo R, Buob D, et al. Bariatric Surgery Reduces Features of Nonalcoholic Steatohepatitis in Morbidly Obese Patients Gastroenterology. 2015;149(2):379-388.

99. Franchello A, Gilbo N, David E, et al. Ischemic preconditioning (IP) of the liver as a safe and protective technique against ischemia/reperfusion injury (IRI). Am J Transplant. 2009;9(7):1629-1639.

100. Degli Esposti D, Sebagh M, Pham P, et al. Ischemic preconditioning induces autophagy and limits necrosis in human recipients of fatty liver grafts, decreasing the incidence of rejection episodes. Cell Death Dis. 2011;2:e111.
101. Chu MJ, Vather R, Hickey AJ, et al. Impact of ischaemic preconditioning on experimental steatotic livers following hepatic ischaemia-reperfusion injury: a systematic review. HPB (Oxford). 2015;17(1):1-10.

102. Patel YA, Berg CL, Moylan CA. Nonalcoholic Fatty Liver Disease: Key Considerations Before and After Liver Transplantation. Dig Dis Sci. 2016;61(5):1406-1416.

103. Beck-Schimmer B, Breitenstein S, Urech S, et al. A randomized controlled trial on pharmacological preconditioning in liver surgery using a volatile anesthetic. Ann Surg. 2008;248(6):909-918.

104. Ben Mosbah I, Rosello-Catafau J, Alfany-Fernandez I, et al. Addition of carvedilol to University Wisconsin solution improves rat steatotic and nonsteatotic liver preservation. Liver Transpl. 2010;16(2):163-171.

105. Nakano H, Nagasaki H, Barama A, et al. The effects of $\mathrm{N}$-acetylcysteine and anti-intercellular adhesion molecule-1 monoclonal antibody against ischemia-reperfusion injury of the rat steatotic liver produced by a choline-methionine-deficient diet. Hepatology. 1997;26(3):670-678.

106. Nativ NI, Maguire TJ, Yarmush G, et al. Liver defatting: an alternative approach to enable steatotic liver transplantation. Am J Transplant. 2012;12(12):3176-3183.

107. Clavien PA, Yadav S, Sindram D, et al. Protective effects of ischemic preconditioning for liver resection performed under inflow occlusion in humans. Ann Surg. 2000;232(2):155-162.

108. Mokuno Y, Berthiaume F, Tompkins RG, et al. Technique for expanding the donor liver pool: heat shock preconditioning in a rat fatty liver model. Liver Transpl. 2004;10(2):264-272.

109. Yamagami K, Enders G, Schauer RJ, et al. Heat-shock preconditioning protects fatty livers in genetically obese Zucker rats from microvascular perfusion failure after ischemia reperfusion. Transpl Int. $2003 ; 16(8): 456-463$

110. Nakamuta M, Morizono S, Soejima $Y$, et al. Short-term intensive treatment for donors with hepatic steatosis in living-donor liver transplantation. Transplantation. 2005;80(5):608-612.

111. Ibrahim ES, Saleh SM, El Hoseeny M, et al. Effect of omega-3 on hepatic regeneration in adult living donors undergoing hepatic resections for liver transplantation: A randomized controlled trial. J Crit Care. 2016;31(1):157-162.

112. Fondevila C, Hessheimer AJ, Ruiz A, et al. Liver transplant using donors after unexpected cardiac death: novel preservation protocol and acceptance criteria. Am J Transplant. 2007;7(7):1849-1855.

113. Dutkowski P, de Rougemont O, Clavien PA. Machine perfusion for marginal liver grafts. Am J Transplant. 2008;8(5):917-924.

114. Jamieson RW, Zilvetti M, Roy D, et al. Hepatic steatosis and normothermic perfusion-preliminary experiments in a porcine model. Transplantation. 2011;92(3):289-295.

115. Nagrath D, Xu H, Tanimura Y, et al. Metabolic preconditioning of donor organs: defatting fatty livers by normothermic perfusion ex vivo. Metab Eng. 2009;11(4-5):274-283. 